Research Article

\title{
Extremal trees for the geometric-arithmetic index with the maximum degree
}

\author{
A. Divya, A. Manimaran* \\ Department of Mathematics, School of Advanced Sciences, Vellore Institute of Technology, Vellore-632014, India
}

(Received: 29 December 2021. Received in revised form: 2 January 2022. Accepted: 15 January 2022. Published online: 27 January 2022.)

(c) 2022 the authors. This is an open access article under the CC BY (International 4.0) license (www.creativecommons.org/licenses/by/4.0/).

\begin{abstract}
For a graph $G$, the geometric-arithmetic index of $G$, denoted by $G A(G)$, is defined as the sum of the quantities $2 \sqrt{d_{x} \times d_{y}} /\left(d_{x}+d_{y}\right)$ over all edges $x y \in E(G)$. Here, $d_{x}$ indicates the vertex degree of $x$. For every tree $T$ of order $n \geq 3$, Vukičević and Furtula [J. Math. Chem. 46 (2009) 1369-1376] demonstrated that $G A(T) \leq \frac{4 \sqrt{2}}{3}+(n-3)$. This result is extended in the present paper. Particularly, for any tree $\mathscr{T}$ of order $n \geq 5$ and maximum degree $\Delta$, it is proved that
\end{abstract}

$$
G A(\mathscr{T}) \leq \begin{cases}2\left(\frac{(2 \Delta-n+1) \sqrt{\Delta}}{\Delta+1}+\frac{(n-\Delta-1) \sqrt{3}}{3}+\frac{(n-\Delta-1) \sqrt{2 \Delta}}{\Delta+2}\right) & \text { if } \Delta>\frac{n-1}{2} \\ 2\left(\frac{\Delta \sqrt{2}}{3}+\frac{\Delta \sqrt{2 \Delta}}{\Delta+2}+\frac{2(n-2 \Delta-1)}{4}\right) & \text { if } \Delta \leq \frac{n-1}{2} .\end{cases}
$$

and the corresponding extremal trees are characterized.

Keywords: topological indices; geometric-arithmetic index; degree of a vertex.

2020 Mathematics Subject Classification: 05C09, 05C92.

\section{Introduction}

Let $G$ be a simple connected graph with the vertex set $V(G)$ and the edges set $E(G)$. The order $n(G)$ of $G$ is the cardinality of the vertex set $V(G)$ and the size $m(G)$ of $G$ is the cardinality of the edge set $E(G)$. The set $N(y)=\{x \in V(G) \mid x y \in E(G)\}$ is the open neighbourhood of a vertex $y \in V(G)$. Also, for a vertex $y \in V(G)$, its degree $d_{y}$ is equal to the cardinality of $N(y)$. The maximum and minimum degrees of a graph $G$ are denoted by $\Delta(G)$ and $\delta(G)$, respectively. A vertex of degree one in a tree is known as a leaf. A vertex adjacent to a leaf is referred to as a stem. A strong stem is a vertex adjacent to two or more leaves. An end stem is a vertex whose only neighbours are leaves, except one. A rooted tree is a directed rooted tree with a distinct vertex $\nu$ (see [9]).

Many degree-based topological indices have been widely studied; for example, see $[5,6,11]$. In this paper, we study one of the degree-based topological indices, namely the geometric-arithmetic index proposed in [12]. The definition of the geometric-arithmetic $(G A)$ index for a simple connected graph $G$ is

$$
G A(G)=\sum_{x y \in E(G)} \frac{2 \sqrt{d_{x} \times d_{y}}}{d_{x}+d_{y}} .
$$

In $[2,14]$, the first three minimum and maximum values of the $G A$ index were determined, and some lower and upper bounds on the $G A$ index for molecular graphs were obtained. Additional details about the mathematical study of the $G A$ index can be found in $[1,3,4,7,10]$.

In [12], Vukičević and Furtula demonstrated the upper bound, given in the next theorem, on the geometric-arithmetic index for trees.

Theorem 1.1. If $T$ is a tree with $n$ vertices, then

$$
G A(T) \leq \frac{4 \sqrt{2}}{3}+(n-3)
$$

where the upper bound is achieved if and only if $T$ is the path graph.

In this work, we extend Theorem 1.1 by providing an upper bound on the geometric-arithmetic index for trees $T$ in terms of order and maximum degree of $T$. We also characterize all the extremal trees attaining the bound.

*Corresponding author (marans2011@gmail.com). 


\section{Some lemmas}

This section gives some lemmas that will be used for proving the main result of this paper. A rooted tree with root $\nu$ is denoted by $\mathscr{T}$, where $\nu$ is a vertex of maximum degree and $\mathscr{N}(\nu)=\left\{v_{1}, v_{2}, \ldots, v_{\Delta}\right\}$. In addition, $g a_{\nu}=E(\mathscr{T}) \rightarrow \mathbb{R}$ is the function defined by $g a_{\nu}(x y)=\frac{\sqrt{d_{x} \times d_{y}}}{d_{x}+d_{y}}$. So, $G A(\mathscr{T})=\sum_{e \in E(\mathscr{T})} g a_{\nu}(e)$.
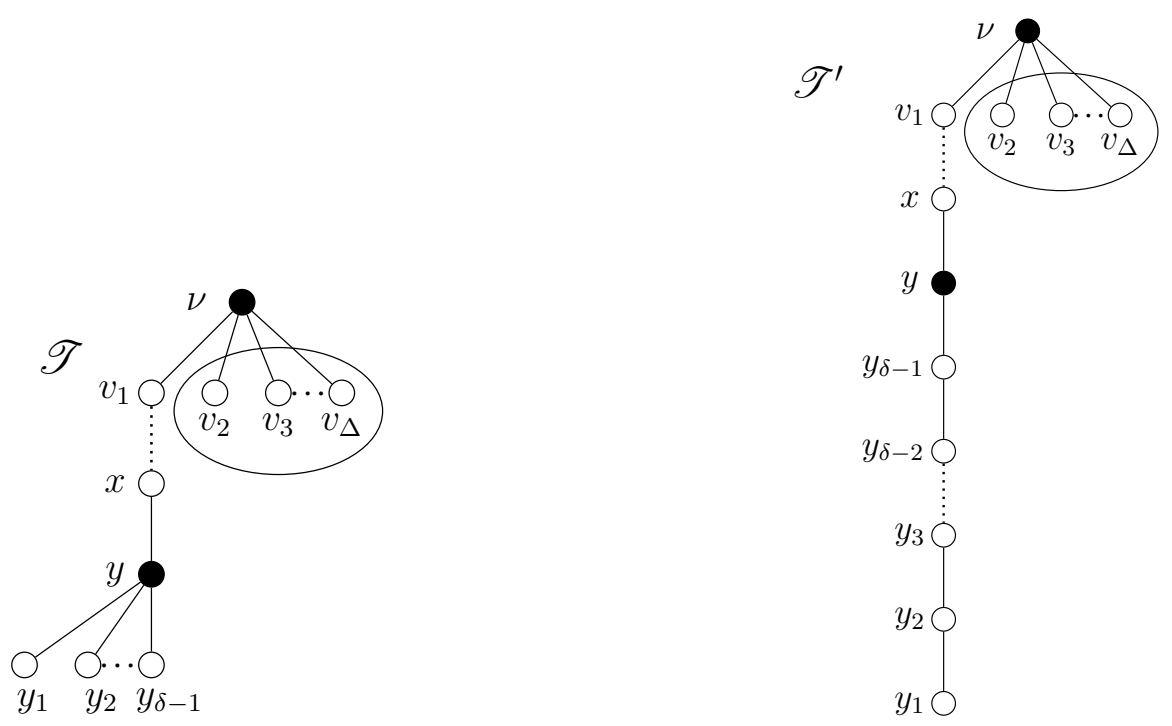

Figure 1: The trees $\mathscr{T}$ and $\mathscr{T}^{\prime}$ used in the proof of Lemma 2.1.

Lemma 2.1. Let $n$ be the order of the tree $\mathscr{T}$ and $\Delta$ be its maximum degree. If $\mathscr{T}$ contains at least one vertex of degree three, an end-stem, and it differs from $\nu$; then there exists a tree $\mathscr{T}^{\prime}$ of order $n$ and maximum degree $\Delta$ such that $G A(\mathscr{T})<G A\left(\mathscr{T}^{\prime}\right)$.

Proof. In $\mathscr{T}$, assume that $y(\neq \nu)$ is an end-stem with $d(y)=\delta \geq 3$ and let $\mathscr{N}(y)=\left\{y_{1}, y_{2}, \ldots, y_{\delta-1}, x\right\}$, where $x$ is the parent of $y$. Take $d(x)=u$ and $S=\left\{y y_{1}, y y_{2}, \ldots, y y_{\delta-1}, y x\right\}$. Let $\mathscr{T}^{\prime}$ be the tree formed by adding the path $y_{\delta-1} y_{\delta-2} \ldots y_{2} y_{1}$ to $\mathscr{T} \backslash\left\{y_{1}, \ldots, y_{\delta-2}\right\}$ (see Figure 1). Obviously, $\mathscr{T}^{\prime}$ is a tree of an order $n$ and $\Delta(\mathscr{T})=\Delta\left(\mathscr{T}^{\prime}\right)$. We have

$$
\begin{aligned}
\frac{1}{2} G A(\mathscr{T}) & =\sum_{x y \notin S} g a_{\nu}(x y)+\sum_{x y \in S} g a_{\nu}(x y) \\
& =\sum_{x y \notin S} g a_{\nu}(x y)+\frac{\sqrt{\delta u}}{\delta+u}+\frac{(\delta-2) \sqrt{\delta}}{\delta+1}+\frac{\sqrt{\delta}}{\delta+1}
\end{aligned}
$$

and

$$
\frac{1}{2} G A\left(\mathscr{T}^{\prime}\right)=\sum_{x y \notin S} g a_{\nu}(x y)+\frac{\sqrt{2 u}}{2+u}+\frac{(\delta-2)}{2}+\frac{\sqrt{2}}{3} .
$$

We derive $G A(\mathscr{T})<G A\left(\mathscr{T}^{\prime}\right)$ by combining (1) and (2), and by using the fact that $\delta \geq 3$.

Lemma 2.2. Let $n$ be the order of the tree $\mathscr{T}$ and $\Delta$ be its maximum degree. If $\mathscr{T}$ contains at least one vertex of degree three, a stem, and it differs from $\nu$; then there exists a tree $\mathscr{T}^{\prime}$ of maximum degree $\Delta$ and order $n$ such that $G A(\mathscr{T})<G A\left(\mathscr{T}^{\prime}\right)$.

Proof. Assume that $x(\neq \nu)$ is a stem of $\mathscr{T}$ with $d(x)=\delta \geq 3$ and let $\mathscr{N}(x)=\left\{x_{1}, x_{2}, \ldots, x_{\delta-1}, y\right\}$, where $x_{1}$ is the parent of $x$ and $d(y)=1$. The vertex $x$ is not an end-stem by Lemma 2.1. Let $\mathscr{T}_{i}$ be the component of $\mathscr{T} \backslash \nu$ that contains $v_{i}$. Let $w$ be the parent of the leaf $q_{i} \in V\left(\mathscr{T}_{i}\right)$ and $q_{i}$ be the maximum distance from $v_{i}$ (see Figure 2). Then, by Lemma 2.1, $d(w)=2$.

Let $\mathscr{T}^{\prime}$ be the tree formed by adding a pendent edge $q_{i} y$ to $\mathscr{T} \backslash x y$ (see Figure 2) and $S=\left\{q_{i} w, x y, x x_{1}, x x_{2}, \ldots, x x_{\delta-1}\right\}$. Obviously, $\mathscr{T}^{\prime}$ is a tree of order $n$ with $\Delta(\mathscr{T})=\Delta\left(\mathscr{T}^{\prime}\right)$. We have

$$
\begin{aligned}
\frac{1}{2} G A(\mathscr{T}) & =\sum_{x y \notin S} g a_{\nu}(x y)+\sum_{x y \in S} g a_{\nu}(x y) \\
& =\sum_{x y \notin S} g a_{\nu}(x y)+\frac{\sqrt{\delta}}{\delta+1}+\sum_{i=1}^{\delta-1} \frac{\sqrt{\delta d_{x_{i}}}}{\delta+d_{x_{i}}}+\frac{\sqrt{2}}{3}
\end{aligned}
$$



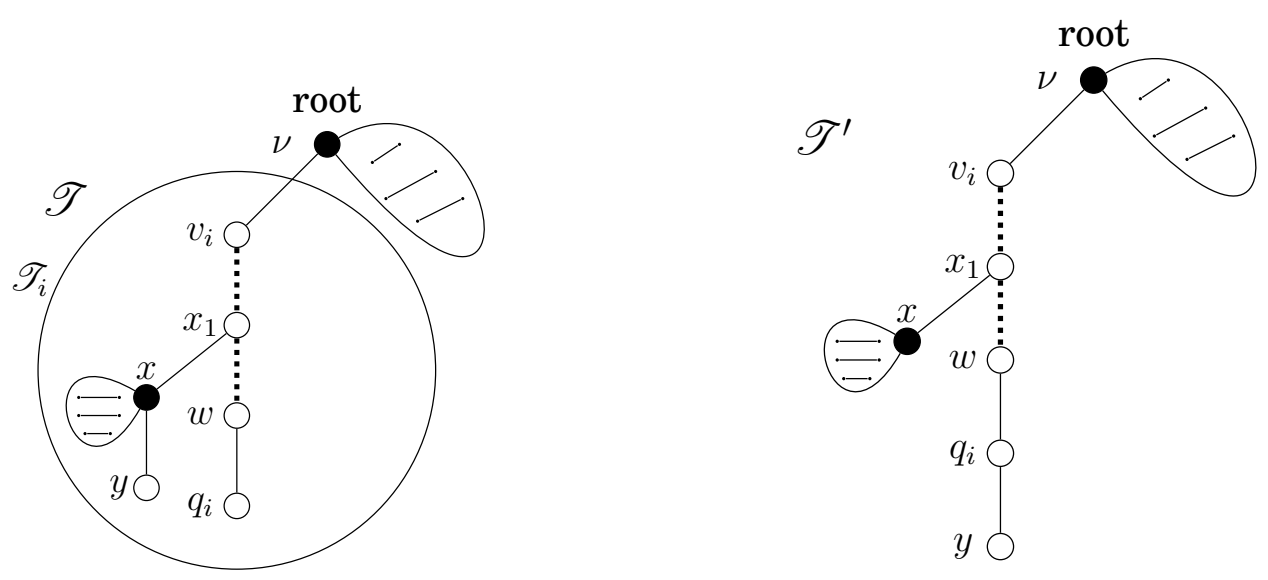

Figure 2: The trees $\mathscr{T}$ and $\mathscr{T}^{\prime}$ used in the proof of Lemma 2.2.

and

$$
\frac{1}{2} G A\left(\mathscr{T}^{\prime}\right)=\sum_{x y \notin S} g a_{\nu}(x y)+\frac{1}{2}+\sum_{i=1}^{\delta-1} \frac{\sqrt{(\delta-1) d_{x_{i}}}}{(\delta-1)+d_{x_{i}}}+\frac{\sqrt{2}}{3} .
$$

We obtain $G A(\mathscr{T})<G A\left(\mathscr{T}^{\prime}\right)$ by combining (3) and (4). This completes the proof.

Lemma 2.3. Let $n$ be the order of the tree $\mathscr{T}$ and $\Delta$ be its maximum degree. If $\mathscr{T}$ contains at least one vertex of degree three and it differs from $\nu$, then there exists a tree $\mathscr{T}^{\prime}$ of maximum degree $\Delta$ and order $n$ such that $G A(\mathscr{T})<G A\left(\mathscr{T}^{\prime}\right)$.

Proof. Let $y \neq \nu$ and $d(y)=\delta \geq 3$, i.e., $d(y, \nu)$ should probably be large. Let $\mathscr{N}(y)=\left\{x_{1}^{0}, x_{2}^{0}, \ldots, x_{\delta-1}^{0}, x_{\delta}\right\}$, where $x_{\delta}$ is the parent of $y$. Let $x_{i}^{0} x_{i}^{1} \ldots x_{i}^{u_{i}}$ be a longest path in $\mathscr{T}_{i}$ starting at $x_{i}^{0}$ for $i=1, \ldots, \delta-1$. Assume that $q_{i} \in V\left(\mathscr{T}_{i}\right)$ is a leaf and $w$ be its parent, such that it has the maximum distance from $v_{i}$ (see Figure 3 ). Suppose that $q_{i} \notin\left\{x_{1}^{u_{1}}, \ldots, x_{\delta-2}^{u_{\delta-2}}\right\}$. We assume that $d(w)=2$, by Lemma 2.1 and 2.2 and by using the option of $y$ and that, apart from leaves, every descendant of $y$ has degree two. We examine two cases.

Case 1. $\delta=3$.

Let $\mathscr{T}^{\prime}$ be the tree formed by adding an edge $q_{i} x_{1}^{0}$ to $\mathscr{T} \backslash y x_{1}^{0}$ (see Figure 3 ) and $S=\left\{y x_{1}^{0}, y x_{2}^{0}, y x_{3}, q_{i} w\right\}$. Obviously, $\mathscr{T}^{\prime}$ is a tree of order $n$ with $\Delta(\mathscr{T})=\Delta\left(\mathscr{T}^{\prime}\right)$. We have

$$
\begin{aligned}
\frac{1}{2} G A(\mathscr{T}) & =\sum_{x y \notin S} g a_{\nu}(x y)+\sum_{x y \in S} g a_{\nu}(x y) \\
& =\sum_{x y \notin S} g a_{\nu}(x y)+\frac{2 \sqrt{6}}{5}+\frac{\sqrt{3 \times d_{x_{3}}}}{3+d_{x_{3}}}+\frac{\sqrt{2}}{3}
\end{aligned}
$$

and

$$
\frac{1}{2} G A\left(\mathscr{T}^{\prime}\right)=\sum_{x y \notin S} g a_{\nu}(x y)+\frac{1}{2}+\frac{1}{2}+\frac{\sqrt{2 \times d_{x_{3}}}}{2+d_{x_{3}}}+\frac{1}{2} .
$$

We obtain $G A(\mathscr{T})<G A\left(\mathscr{T}^{\prime}\right)$ by combining (5) and (6).

Case 2. $\delta \geq 4$.

Let $q_{i} \neq x_{\delta-1}^{u_{\delta-1}}$. Let $\mathscr{T}^{\prime}$ be the tree formed by adding edges $x_{1}^{0} q_{i}, x_{2}^{0} x_{1}^{u_{1}}, x_{3}^{0} x_{2}^{u_{2}}, \ldots, x_{\delta-1}^{0} x_{\delta-2}^{u_{\delta-2}}$ to $\mathscr{T} \backslash x_{1}^{0} y, x_{2}^{0} y, \ldots, x_{\delta-1}^{0} y$. Clearly, $\mathscr{T}^{\prime}$ is a tree of order $n$ and $\Delta(\mathscr{T})=\Delta\left(\mathscr{T}^{\prime}\right)$. Let $S=\left\{q_{i} w, y x_{\delta}\right\} \cup\left\{y x_{i}^{0}, x_{i}^{u_{i}} x_{i}^{u_{i}-1} \mid 1 \leq i \leq \delta-1\right\}$. By definition, we have

$$
\begin{aligned}
\frac{1}{2} G A(\mathscr{T}) & =\sum_{x y \notin S} g a_{\nu}(x y)+\sum_{x y \in S} g a_{\nu}(x y) \\
& =\sum_{x y \notin S} g a_{\nu}(x y)+\frac{(\delta-1) \sqrt{2}}{3}+\frac{(\delta-1) \sqrt{2 \delta}}{\delta+2}+\frac{\sqrt{\delta \times d_{x_{\delta}}}}{\delta+d_{x_{\delta}}}+\frac{\sqrt{2}}{3}
\end{aligned}
$$

and

$$
\frac{1}{2} G A\left(\mathscr{T}^{\prime}\right)=\sum_{x y \notin S} g a_{\nu}(x y)+\frac{2(\delta-1)}{4}+\frac{\sqrt{2}}{3}+\frac{2(\delta-1)}{4}+\frac{\sqrt{d_{x_{\delta}}}}{1+d_{x_{\delta}}}
$$


From (7) and (8), we obtain $G A(\mathscr{T})<G A\left(\mathscr{T}^{\prime}\right)$.

Next, let $q_{i}=x_{\delta-1}^{u_{\delta-1}}$. Let $\mathscr{T}^{\prime}$ be the tree formed by adding edges $x_{1}^{0} q_{i}, x_{i}^{u_{i}} x_{i+1}^{0}$ for $1 \leq i \leq \delta-3$ to $\mathscr{T} \backslash y x_{i}^{0}$ for $i=1, \ldots, \delta-2$. Assume that $S=\left\{y x_{\delta}\right\} \cup\left\{y x_{i}^{0}, x_{i}^{u_{i}} x_{i}^{u_{i}-1} \mid 1 \leq i \leq \delta-1\right\}$. By definition, we have

$$
\begin{aligned}
\frac{1}{2} G A(\mathscr{T}) & =\sum_{x y \notin S} g a_{\nu}(x y)+\sum_{x y \in S} g a_{\nu}(x y) \\
& =\sum_{x y \notin S} g a_{\nu}(x y)+\frac{(\delta-1) \sqrt{2}}{3}+\frac{(\delta-1) \sqrt{2 \delta}}{\delta+2}+\frac{\sqrt{\delta \times d_{x_{\delta}}}}{\delta+d_{x_{\delta}}}
\end{aligned}
$$

and

$$
\frac{1}{2} G A\left(\mathscr{T}^{\prime}\right)=\sum_{x y \notin S} g a_{\nu}(x y)+\frac{\delta-2}{2}+\frac{\sqrt{2}}{3}+\frac{\delta-1}{2}+\frac{\sqrt{2 \times d_{x_{\delta}}}}{2+d_{x_{\delta}}} .
$$

Note that

$$
\begin{gathered}
\frac{\sqrt{\delta \times d_{u_{\delta}}}}{\delta+d_{u_{\delta}}}<\frac{\sqrt{2 \times d_{u_{\delta}}}}{2+d_{u_{\delta}}} \\
\frac{(\delta-2) \sqrt{2}}{3}+\frac{(\delta-1) \sqrt{2 \delta}}{\delta+2} \leq \frac{(\delta-3) \sqrt{2 \delta}+2 \sqrt{2}}{2}, \\
\frac{\delta-2}{2}+\frac{\sqrt{2}}{3}+\frac{\delta-1}{2}=\frac{(\delta-3) \sqrt{2 \delta}+2 \sqrt{2}}{2}+\frac{1}{7} .
\end{gathered}
$$

Thus, from (9) and (10) we conclude that $G A(\mathscr{T})<G A\left(\mathscr{T}^{\prime}\right)$.
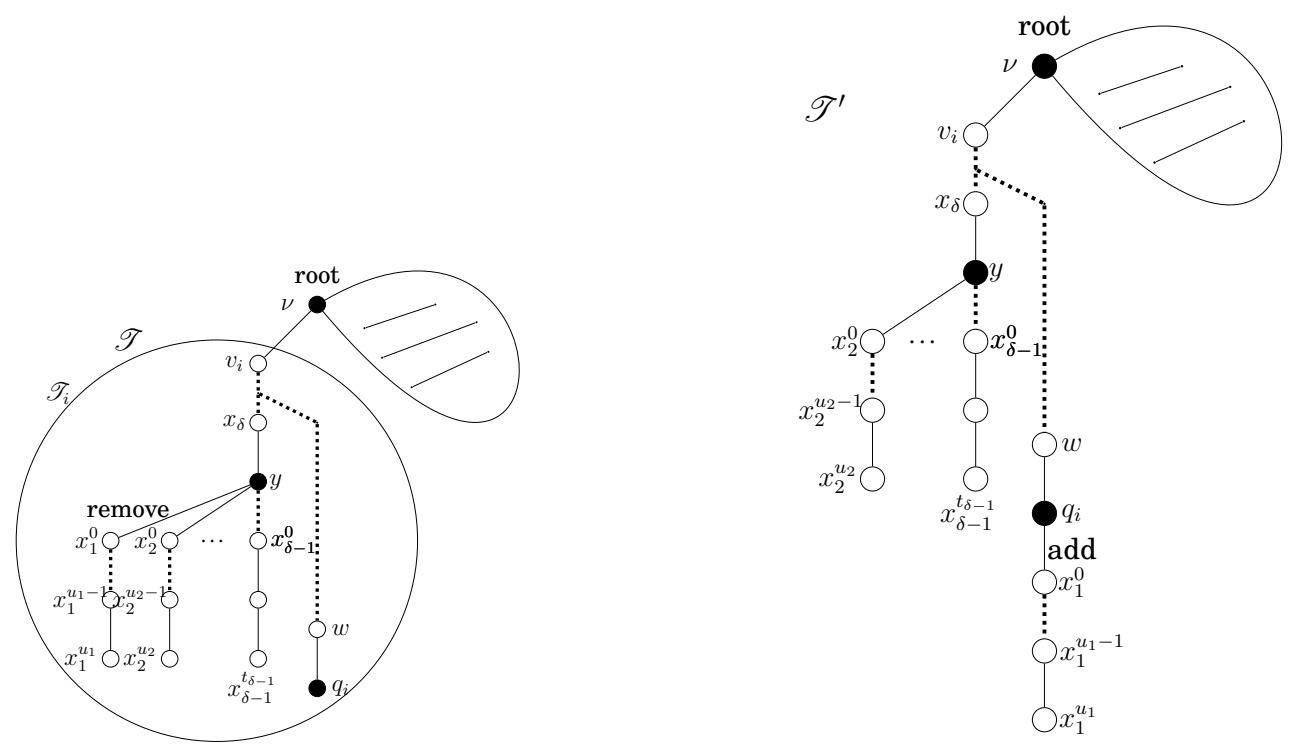

Figure 3: The trees $\mathscr{T}$ and $\mathscr{T}^{\prime}$ used in the proof of Lemma 2.3.

A spider tree (also known as a star-like tree) $[8,13]$ has at most one vertex (known as the center) of degree greater than two; see [9] for more information. A leg of a spider tree is a path that connects its center to a vertex of degree one.

Lemma 2.4. Let $n$ be the order of the spider tree $\mathscr{T}$ with $k \geq 3$ legs. If $\mathscr{T}$ has a leg of length one and a leg of length at least 3 , then there exists a spider tree $\mathscr{T}^{\prime}$ of order $n$ with $k$ legs such that $G A(\mathscr{T})<G A\left(\mathscr{T}^{\prime}\right)$.

Proof. Let the center of $\mathscr{T}$ be $\nu$ and $\mathscr{N}(\nu)=\left\{v_{1}, v_{2}, \ldots, v_{k}\right\}$. Let $\nu$ be the root of $\mathscr{T}$. Let $d\left(v_{1}\right)=1$, without loss of generality, and let the leg $v_{k} z_{1} z_{2} \ldots z_{u}$ be a longest one in $\mathscr{T}$. Let the tree $\mathscr{T}^{\prime}$ be formed by adding a pendent edge $v_{1} z_{u}$ to $\mathscr{T} \backslash z_{u} z_{u-1}$. Assume that $S=\left\{v_{1} \nu, z_{u} z_{u-1}, z_{u-2} z_{u-1}\right\}$. By definition, we have

$$
\frac{1}{2} G A(\mathscr{T})=\sum_{x y \notin S} g a_{\nu}(x y)+\sum_{x y \in S} g a_{\nu}(x y)
$$




$$
=\sum_{x y \notin S} g a_{\nu}(x y)+\frac{2}{k+1}+\frac{\sqrt{2}}{3}+\frac{\sqrt{3}}{4}
$$

and

$$
\frac{1}{2} G A\left(\mathscr{T}^{\prime}\right)=\sum_{x y \notin S} g a_{\nu}(x y)+\frac{\sqrt{2 k}}{k+2}+\frac{\sqrt{2}}{3}+\frac{\sqrt{2}}{3} .
$$

From (11) and (12), we see that $G A(\mathscr{T})<G A\left(\mathscr{T}^{\prime}\right)$.

\section{Main result}

In this section, we present the main result of this paper. Figure 4 represents trees with the maximum $G A$ index among trees of maximum degree 4 and order $n$ for $n=7,9,10$.

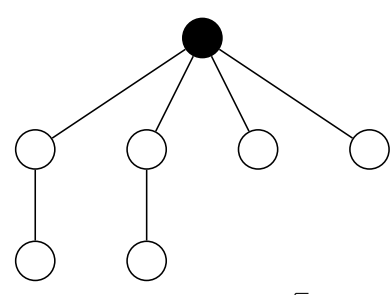

(a) $G A\left(\mathscr{T}_{7}\right)=\frac{40 \sqrt{2}+24}{15}$

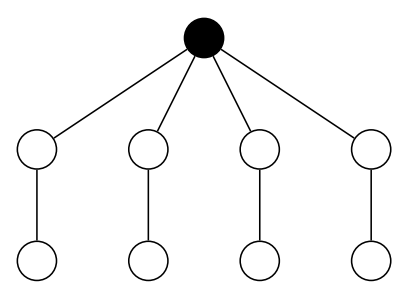

(b) $G A\left(\mathscr{T}_{9}\right)=\frac{16 \sqrt{2}}{3}$

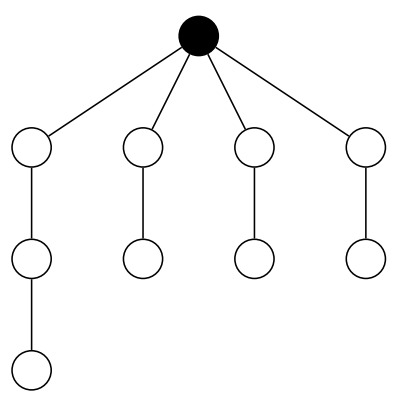

(c) $G A\left(\mathscr{T}_{10}\right)=\frac{64 \sqrt{2}+12}{12}$

Figure 4: Trees attaining the maximum $G A$ index among trees of maximum degree 4 and orders 7,9 , and 10.

Theorem 3.1. If $\mathscr{T}$ is a tree of order $n \geq 5$ and maximum degree $\Delta$ then

$$
G A(\mathscr{T}) \leq \begin{cases}2\left(\frac{(2 \Delta-n+1) \sqrt{\Delta}}{\Delta+1}+\frac{(n-\Delta-1) \sqrt{3}}{3}+\frac{(n-\Delta-1) \sqrt{2 \Delta}}{\Delta+2}\right) & \text { if } \Delta>\frac{n-1}{2} \\ 2\left(\frac{\Delta \sqrt{2}}{3}+\frac{\Delta \sqrt{2 \Delta}}{\Delta+2}+\frac{2(n-2 \Delta-1)}{4}\right) & \text { if } \Delta \leq \frac{n-1}{2}\end{cases}
$$

with equality if and only if $\mathscr{T}$ is a spider tree and all of its legs have lengths at most two or all of its legs have lengths at least two.

Proof. Let $G A\left(\mathscr{T}_{1}\right)=\max \{G A(\mathscr{T}) \mid \mathscr{T}$ be a tree of order $n$ and maximum degree $\Delta\}$. Let $\nu$ be the root of $\mathscr{T}_{1}$ and its degree be $\Delta$. If $\Delta=2$, then $\mathscr{T}$ is the path of order $n$, and the result follows from Theorem 1.1. Next, we assume that $\Delta \geq 3$. By using Lemmas 2.1, 2.2 and 2.3, we conclude that $\mathscr{T}_{1}$ is a spider tree with center $\nu$. By Lemma 2.4, all the legs of $\mathscr{T}_{1}$ have lengths either at most two or at least two.

Suppose that all the legs of $\mathscr{T}_{1}$ have lengths at least two. Then, it is obvious that $\Delta \leq \frac{n-1}{2}$ and

$$
G A\left(\mathscr{T}_{1}\right)=2\left(\frac{\Delta \sqrt{2}}{3}+\frac{\Delta \sqrt{2 \Delta}}{\Delta+2}+\frac{(n-2 \Delta-1)}{2}\right) .
$$

Next, assume that all the legs of $\mathscr{T}_{1}$ have lengths at most two. Suppose $\mathscr{T}_{1}$ has at least one leg of length one. The result is immediate if $\mathscr{T}_{1}$ is a star graph. If $\mathscr{T}_{1}$ is not a star, then $(2 \Delta+1-n) \sqrt{\Delta}$ is the number of leaves adjacent to $\nu$ and thence

$$
G A(T)=2\left(\frac{(2 \Delta+1-n) \sqrt{\Delta}}{\Delta+1}+\frac{(n-\Delta-1) \sqrt{3}}{3}+\frac{(n-\Delta-1) \sqrt{2 \Delta}}{\Delta+2}\right) .
$$

The proof is completed.

\section{References}

[1] K. C. Das, On geometric-arithmetic index of graphs, MATCH Commun. Math. Comput. Chem. 64 (2010) 619-630.

[2] K. C. Das, I. Gutman, B. Furtula, Survey on geometric-arithmetic indices of graphs, MATCH Commun. Math. Comput. Chem. 65 (2011) 595-644.

[3] K. C. Das, I. Gutman, B. Furtula, On first geometric-arithmetic index of graphs, Discrete Appl. Math. 159 (2011) $2030-2037$.

[4] K. C. Das, N. Trinajstić, Comparison between geometric-arithmetic indices, Croat. Chem. Acta 85 (2012) 353-357. 
[5] B. Furtula, I. Gutman, M. Dehmer, On structure-sensitivity of degree-based topological indices, Appl. Math. Comput. 219 (2013) 8973-8978.

[6] I. Gutman, Degree-based topological indices, Croat. Chem. Acta 86 (2013) 351-361.

[7] M. Mogharrab, G. H. Fath-Tabar, Some bounds on GA index of graphs, MATCH Commun. Math. Comput. Chem. 65 (2010) 33-38.

[8] J. Rada, O. Araujo, Higher order connectivity index of starlike trees, Discrete Appl. Math. 119 (2002) 287-295.

[9] R. Rasi, S. M. Sheikholeslami, I. Gutman, On harmonic index of trees, MATCH Commun. Math. Comput. Chem. 78 (2017) $405-416$.

[10] J. M. Rodríguez, J. M. Sigarreta, On the geometric-arithmetic index, MATCH Commun. Math. Comput. Chem. 74 (2015) 103-120.

[11] N. Trinajstić, Chemical Graph Theory, CRC Press, Boca Raton, 1992.

[12] D. Vukičević, B. Furtula, Topological index based on the ratios of geometrical and arithmetical means of end-vertex degrees of edges, J. Math. Chem. 46 (2009) 1369-1376.

[13] M. Watanabe, A. J. Schwenk, Integral starlike trees, J. Austral. Math. Soc. 28 (1979) 120-128.

[14] Y. Yuan, B. Zhou, N. Trinajstić, On geometric-arithmetic index, J. Math. Chem. 47 (2010) 833-841. 